

\title{
Molecular Motions in Thermotropic Liquid Crystals Studied by NMR Spin-Lattice Relaxation
}

\author{
R.C. Zamar, C.E. González and O. Mensio \\ Facultad de Matemática, Astronomía y Física, \\ Universidad Nacional de Córdoba \\ Ciudad Universitaria, 5000 Córdoba, Argentina
}

Received 29 October, 1998

\begin{abstract}
Nuclear magnetic resonance relaxation experiments with field cycling techniques proved to be a valuable tool for studying molecular motions in liquid crystals, allowing a very broad Larmor frequency variation, sufficient to separate the cooperative motions from the liquidlike molecular diffusion. In new experiments combining NMR field cycling with the JeenerBroekaert order-transfer pulse sequence, it is possible to measure the dipolar order relaxation time $\left(T_{1 D}\right)$, in addition to the conventional Zeeman relaxation time $\left(T_{1 Z}\right)$ in a frequency range of several decades. When applying this technique to nematic thermotropic liquid crystals, $T_{1 D}$ showed to depend almost exclusively on the order fluctuation of the director mechanism in the whole frequency range. This unique characteristic of $T_{1 D}$ makes dipolar order relaxation experiments specially useful for studying the frequency and temperature dependence of the spectral properties of the collective motions.
\end{abstract}

\section{Introduction}

Liquid crystal molecules when ordered in a mesophase undergo different kinds of motions. Some of them are individual molecular motions, like anisotropic rotational and translational diffusion. These are liquidlike rapid random jumps which can be characterized with a correlation time $\tau_{c}$, generally falling in the range $10^{-8}-10^{-11} s$. There are also slower reorientational motions involving a large number of molecules, that are characteristic of the liquid crystalline states. These collective molecular fluctuations, called order fluctuations of the director (OFD), are described through a superposition of a broad spectrum of individual purely dissipative modes, with relaxation times ranging from $10^{-4}$ to $10^{-9} \mathrm{~s}$. As a consequence of the diversity of characteristic times of the fluctuations in liquid crystals, the different processes are clearly visible in different time scales. For example, in Zeeman order relaxation NMR experiments, the local motions dominate in high frequencies $\left(10^{6}-10^{8} \mathrm{~Hz}\right)$ while the order fluctuations represent the main mechanism of spin relaxation in the low and medium ranges $\left(10^{3}-10^{6} \mathrm{~Hz}\right)$.
The NMR relaxation method has been used for many years for studying the complex anisotropic reorientation of molecules in the liquid-crystalline state. The measured quantities are spin-lattice relaxation rates which depend on the intra- and inter-molecular interactions of the nuclear spin magnetization with local magnetic and electric fields; these fields being modulated by the underlying molecular motions.[1] It is experimentally observed that in conventional liquids the relaxation times are almost independent of the Larmor frequency. On the contrary, in liquid crystal mesophases, the presence of cooperative motions causes the relaxation times to have a marked frequency dependence, showing usually a dispersion of about two orders of magnitude in the frequency range $10^{4}$ to $10^{8} \mathrm{~Hz}$.

NMR studies of molecular motions in liquid crystals are presently usually performed by essentially two different spin-relaxation experiments which can provide a satisfactory number of data to disentangle the underlying spectral densities of the superimposed molecular reorientation: On the one hand by combining highfield, Zeeman relaxation $\left(T_{1 Z}\right)$ with high-field dipolar and quadrupolar order relaxation $\left(T_{1 D}\right.$ and $T_{1 Q}$ respec- 
tively) measurements at constant Larmor frequency $\nu_{L}$, in ${ }^{1} \mathrm{H}$ or ${ }^{2} \mathrm{H}$ as a function of temperature.[2] On the other hand by frequency dependent $T_{1 Z}$ studies over a broad Larmor frequency, $\nu$, range, often applying fastfield cycling (FC) techniques.[3] Now, with adequate instruments such frequency dependent measurements are not necessarily restricted to $T_{1 Z}$, but in principle are also applicable to $T_{1 D}$; this however has only been tried rarely and not systematically, mainly because of experimental limitations.

Due to the complexity of the molecular motions, and the fact that the diffusion motions make their main contribution in high frequencies, it is difficult to distinguish between the cooperative and the local motions from standard high field NMR relaxation measurements. However, by combining field cycling data supplemented with conventional measurements (for the highest frequencies), it is possible to cover a broad frequency range $\left(10^{3}-10^{8} \mathrm{~Hz}\right)$, allowing to distinguish the mechanisms unambiguously.

The basic theory for explaining relaxation experiments relates the relaxation times with the spectral densities of the lattice; that is, with the nonspin variables. The second step demands a model for the spectral densities that relates them with the particular system (viscosity, elastic constants, etc) and with the externally controlled variables (temperature, frequency, orientation). The theory of spin-lattice relaxation for liquid crystals is based on the weak-order assumption, $[1,4,5]$ that was traditionally applied in liquids and solids. The starting point is the stochastic Liouville equation for the density operator of the spin system, which is integrated up to second order in the perturbation (the interaction with the lattice). The spin system is considered as an ensemble of isolated spins, the neighboring spins being considered as far as they form part of the lattice.

In the calculation of the spectral densities of the lattice, the molecular motions are generally modeled by stochastic Markovian processes. This is suitable for liquid crystals, because of the low degree of positional order of the molecular motions. The models for rotational and traslational diffusion are basically the same as those used in liquids, with some refinements to reflect the characteristic anisotropy of the liquid crystals. $[6,7,8]$ The models for the OFD are based on the hydrodynamic theory.[9] It is assumed that the molecules follow the director dynamics; so doing the effects of the intermolecular short range correlation are neglected.[10, 11] This approach yields spectral densities proportional to $\nu^{-1 / 2}$ and $\nu^{-1}$ for nematics $[12,13]$ and smectics $[14,15]$ respectively. In the case of nematics, this law has been confirmed by field cycling experiments of Zeeman relaxation of protons and deuterons in numerous nematogens.[3, 16] It was observed that the OFD are dominant in the frequency range $10^{3}$ $5 \times 10^{5} \mathrm{~Hz}$. There are also clear NMR FC examples of both smectic thermotropic phases and smectic-type lyotropics displaying the mentioned linear frequency dependence.[18, 19, 20, 21]

One of the main hypotheses of the traditional theory of relaxation concerning the correlation time of the fluctuations is that of the fast motion: $\nu_{S I} \tau_{c}<1$ where $\nu_{S I}$ is the characteristic frequency of the spin interaction (dipolar or quadrupolar) and $\tau_{C}$ is the correlation time of the fluctuation. A consequence of this assumption is that the spin interactions can be neglected from the spin dynamics in the microscopic time scale. [5] Though the former condition is not fulfilled by the slowest components of the OFD, this fact does not constitute a difficulty in high field Zeeman order relaxation. In these cases the picture of a single spin interacting with a random bath is adequate, hence the details of the spin interactions during the lifetime of the fluctuations can be neglected. The analysis of experimental $T_{1 Z}$ within this theoretical framework showed to be consistent. [2, 3, 22]

However, this would not be the case of dipolar order relaxation, where the relaxing magnitude is the dipole-dipole energy. In fact, recent results of $T_{1 D}$ in thermotropic nematic liquid crystals, as a function of the Larmor frequency in field cycling experiments evidenced clear discrepancies with the standard weakorder theory [28]. The OFD are the leading relaxation mechanisms even at high frequencies, having a larger weight than the predicted by the standard approach. This fact indicates the presence of additional relaxation mechanisms, driven by the OFD, which are not taken into account by the traditional approach. Due to its higher sensitivity to the cooperative motions, $T_{1 D}$ is a powerful tool for investigating spectral properties of 
hydrodynamic fluctuations in mesophases.

The possibility of measuring several independent relaxation times, reflecting different features of the molecular motions, together with the existence of powerful experimental techniques, make NMR relaxation a very important tool for characterizing molecular motions in mesophases.

In Section 2 we present a short review of the theoretical background necessary to interpret the experimental results. Some examples illustrating the possibilities of field cycling NMR experiments, in protons and deuterons, are shown in Section 3. This selection does not attempt to be a review of this vast field. Finally in Section 4 some new, controversial results on dipolar order relaxation are presented, and discussed in terms of the existent theory, together with an analysis of a possible way to understand the disagreement.

\section{Theoretical background}

NMR relaxation experiments deal with the nuclear spin degrees of freedom and provide information on the host lattice through the time evolution of the spin system. It is usual to think on the spin system as a thermodynamic system in contact with a reservoir (the lattice) having a huge number of degrees of freedom. In a typical experiment, energy in the form of radio frequency pulses, is delivered to the spins in order to prepare it

$$
\begin{aligned}
& A_{l k}^{0}=-\gamma \hbar\left\{-I_{l}^{z} I_{k}^{z}+\frac{1}{4}\left(I_{l}^{+} I_{k}^{-}+I_{k}^{+} I_{l}^{-}\right)\right\} \\
& A_{l k}^{ \pm 1}=-\frac{3}{2} \gamma \hbar\left(I_{l}^{z} I_{k}^{ \pm}+I_{l}^{ \pm} I_{k}^{z}\right) \\
& A_{l k}^{ \pm 2}=-\frac{3}{4} \gamma \hbar I_{l}^{ \pm} I_{k}^{ \pm}
\end{aligned}
$$

$\theta$ and $\phi$ are the spherical angular coordinates of the internuclear vector $\mathbf{r}_{l k}$ respect to a frame whose $z$-axis is parallel to the magnetic field $\mathbf{H}_{\mathbf{0}}$. In the quadrupolar case the interaction is that of the nuclear quadrupolar moment with the electric field gradient of the electronic charge distribution. The angular dependence refers to the orientation of the principal axis system of the electric field gradient tensor with respect to the laboratory frame.[2] (in the quantum mechanical sense). The system is then allowed to develop in contact with the lattice during the evolution period. The last step of the experiment is just to pick up the result of the evolution. The recovery of the equilibrium state occurs with a characteristic time called spin-relaxation time, conditioned by the efficiency of the spin-lattice relaxation mechanisms.

The Hamiltonian describing the spin system and the lattice is

$$
\mathcal{H}=\mathcal{H}_{S}+\mathcal{H}_{L}+\mathcal{H}_{S L}
$$

$\mathcal{H}_{S}$ and $\mathcal{H}_{L}$ are the spin and the lattice Hamiltonians respectively, and $\mathcal{H}_{S L}$ represents the spin-lattice interaction.[1] The spin Hamiltonian contains the energy of interaction with the external magnetic field (Zeeman energy) and the average energy of the spin interactions. For the usually available magnetic fields, the principal spin interactions are the dipole-dipole and quadrupolar energies. For example, in the case of the dipolar interaction between like spins the spin Hamiltonian is:

$$
\begin{aligned}
\mathcal{H}_{S} & =\mathcal{H}_{Z}+\overline{\mathcal{H}_{\mathcal{S I}}} \quad \text { with } \\
\mathcal{H}_{Z} & =-\gamma \hbar H_{0} \sum_{j} I_{j z}, \quad \text { and } \\
\overline{\mathcal{H}_{S \mathcal{I}}} & =\frac{1}{2} \sum_{l k} \sum_{q} A_{l k}^{q} \overline{F_{l k}^{q}}
\end{aligned}
$$

$\gamma$ is the gyromagnetic ratio and the upper bar means average over the lattice ensemble. $A_{l k}^{q}$ and $F_{l k}^{q}$ are spin and lattice operators associated to the dipolar spin interaction:

$$
\begin{aligned}
& F_{l k}^{0}=\frac{1}{r_{l k}^{3}}\left(1-3 \cos ^{2} \theta_{l k}\right) \\
& F_{l k}^{ \pm 1}=\frac{1}{r_{l k}^{3}} \sin \theta_{l k} \cos \theta_{l k} e^{ \pm i \phi_{l k}} \\
& F_{l k}^{ \pm 2}=\frac{1}{r_{l k}^{3}} \sin ^{2} \theta_{l k} e^{ \pm 2 i \phi_{l k}} .
\end{aligned}
$$

The spin-lattice interaction Hamiltonian reflects the fluctuations of the spin interactions caused by the lattice motions. For example, for dipolar interaction:

$$
\mathcal{H}_{S L}=\Delta \mathcal{H}_{D}=\frac{1}{2} \sum_{l k} \sum_{q=0 \pm 1 \pm 2} A_{l k}^{q} \Delta F_{l k}^{q}
$$

where: $\Delta F_{l k}^{q}(t) \equiv F_{l k}^{q}(t)-\overline{F_{l k}^{q}}$.

The density operator of the whole system (spins and 
lattice) satisfies the Liouville equation

$$
\frac{d \rho}{d t}=-\frac{i}{\hbar}[\mathcal{H}, \rho]
$$

In NMR experiments only the spin system is detected, therefore, the relevant statistical operator for calculating the time evolution of the observables associated to the spin system is the reduced density operator $\sigma \equiv \operatorname{Tr}_{L}\{\rho\}$, where $\operatorname{Tr}_{L}$ stands for the trace over the lattice variables. The time evolution of this operator is described by [23]

$$
\frac{d \sigma}{d t}=-\frac{i}{\hbar} \operatorname{Tr}_{L}[\mathcal{H}, \rho]
$$

At this point two basic assumptions are made: i) The coupling between the spin system and the lattice is weak enough for allowing to factorize the total density operator in the second member of Eq.(7) as a first approximation.

ii) The lattice is considered as a quantum dissipative system having so many degrees of freedom that dissipates the energy transferred by the spins in a short time of the order of the lattice correlation time, which is much shorter than the interval where the observed magnitude varies appreciably. This means that the lattice can be considered always at the equilibrium state. Under these conditions, the master equation in the interaction picture adopts the typical form of Markovian processes: $\frac{d \sigma^{*}(t)}{d t}$ depends only on $\sigma^{*}(t)$, with time independent coefficients: $[1,23]$

$$
\frac{d \sigma^{*}(t)}{d t}=-\hbar^{-2} \int_{0}^{\infty} d t^{\prime} \operatorname{Tr}_{L}\left[\mathcal{H}_{S L}^{*}(t),\left[\mathcal{H}_{S L}^{*}\left(t^{\prime}\right), \sigma^{*}(t) \rho_{L}(0)\right]\right]
$$

where $\rho_{L}(0)$ is the equilibrium canonical density operator of the lattice and '*' indicates that operators are expressed in the interaction picture.

Eq.(7) can be rewritten in a more convenient form for studying longitudinal relaxation. Following Abragam [1]

$$
\begin{aligned}
\frac{d \sigma^{*}(t)}{d t}= & -\frac{\hbar^{-2}}{2} \operatorname{Tr}_{L}\left\{\int_{-\infty}^{\infty} d t^{\prime}\left[\mathcal{H}_{S L}^{*}(t),\left[\mathcal{H}_{S L}^{*}\left(t^{\prime}\right), \sigma^{*}(t)\right]\right] \rho_{L}(0)-\right. \\
& -\int_{-\infty}^{\infty} d t^{\prime}\left[\mathcal{H}_{S L}^{*}\left(t^{\prime}\right), \rho_{L}(0)\right] \sigma^{*}(t) \mathcal{H}_{S L}^{*}(t)+ \\
& \left.+\mathcal{H}_{S L}^{*}(t) \sigma^{*}(t) \int_{-\infty}^{\infty} d t^{\prime}\left[\mathcal{H}_{S L}^{*}\left(t^{\prime}\right), \rho_{L}(0)\right]\right\}
\end{aligned}
$$

The first term has the structure of an ensemble average over the the lattice variables and coincides formally with the semiclassical master equation as deduced from the stochastic Liouville equation. The traditional weak order master equation is obtained from Eq.(8) by assuming high lattice temperature $\left(h \nu / K_{B} T \ll 1\right)$, and that the spin system is never very remote from a state with equal populations of all spin energy levels ( $\sigma^{*} \simeq \mathrm{I} / A$, I being the identity operator in the Hilbert space of the spin system and $A$ being the number of degrees of freedom of the spin system).[1, 24] The resulting equation coincides essentially with the first term of Eq.(8) but including the correct stationary state:

$$
\frac{d \sigma^{*}(t)}{d t}=-\frac{\hbar^{-2}}{2} \int_{-\infty}^{\infty} d t^{\prime} \overline{\left[\mathcal{H}_{S L}^{*}(t),\left[\mathcal{H}_{S L}^{*}\left(t^{\prime}\right), \sigma^{*}(t)-\sigma_{0}\right]\right.}
$$


This key equation allows the calculation of the time dependence of any observable of the spin system $\frac{d\left\langle\mathcal{O}^{*}\right\rangle}{d t}=\operatorname{Tr}_{s}\left[\frac{d \sigma^{*}(t)}{d t} \mathcal{O}^{*}(t)\right]$. It describes the irreversible evolution of a macroscopic quantity and allows the calculation of the transport coefficients. In NMR relaxation such coefficients are the relaxation times; they can be related to the spectral densities, that is the Fourier transforms of the time correlation functions of the fluctuating lattice operators, evaluated at the Larmor frequency, $\nu$. For example, the time evolution of the $z$-component of the magnetization (parallel to the external field), due to dipolar coupling in a system of two like spins is

$$
\frac{d}{d t}\left\langle I_{z}+I_{z}^{\prime}\right\rangle=\frac{1}{T_{1 Z}}\left\{\left\langle I_{z}+I_{z}^{\prime}\right\rangle-\left\langle I_{z}+I_{z}^{\prime}\right\rangle_{e q u i l}\right\}
$$

where

$$
T_{1 Z}^{-1}=\frac{9}{8} \gamma^{4} \hbar^{2}\left\{J^{1}(\omega)+J^{2}(2 \omega)\right\}
$$

is the Zeeman order relaxation time and $\omega=2 \pi \nu$. The spectral densities $J^{q}(\omega)$ are defined as

$$
J^{q}(\omega)=\int_{-\infty}^{\infty} \overline{F^{q} F^{-q}(t)} \exp ^{i q \omega t} d t .
$$

In Table 1 we summarize the standard theoretical expressions for the most commonly measured relaxation times. There we show the observable corresponding to each case, the kind of fluctuations responsible for dissipating the initial condition, and the relaxation times formulae in terms of the spectral densities. These expressions are the outcome of the traditional weak order theory [Eq.(9)].

Table I: Relaxation rates within the weak order theory of spin relaxation. In the three first cases the observable is the energy of interaction of isolated spins. For $T_{1 D}^{-1}$ the observable is the secular dipole-dipole energy, and a model of isolated spin pairs is assumed. Here $C_{D}=\frac{9}{8} \gamma^{4} \hbar^{2}, C_{Q}=\frac{3}{2}\left(\frac{e^{2} q Q}{\hbar}\right)^{2}$, and $Q$ is the nuclear quadrupole moment.

\begin{tabular}{|ll|ll|c|c|}
\hline & Observable & Relaxation time & SL interaction & Refs. \\
\hline$\sum\left\langle I_{z j}\right\rangle$ & proton Zeeman order & $T_{1 Z}^{-1}=C_{D} \sum_{l k} J_{l k}^{(1)}(\omega)+J_{l k}^{(2)}(2 \omega)$ & dipolar & {$[1,4]$} \\
$\left\langle I_{z}\right\rangle$ & deut. Zeeman order & $T_{1 Z}^{-1}=C_{Q}\left[J^{(1)}(\omega)+4 J^{(2)}(2 \omega)\right]$ & quadrupolar & {$[2]$} \\
$\left\langle\mathcal{\mathcal { H }}_{Q}^{0}\right\rangle$ & quadrupolar order & $T_{1 Q}^{-1}=3 C_{Q} J^{(1)}(\omega)$ & quadrupolar & {$[25,26]$} \\
$\left\langle\mathcal{\mathcal { H }}_{D}^{0}\right\rangle$ & dipolar order & $T_{1 D}^{-1}=3 C_{D} J^{(1)}(\omega)$ & dipolar & {$[33,50]$} \\
\hline
\end{tabular}

The formula for proton Zeeman relaxation is a direct generalization of Eq.(10) for the case of many noninteracting spins [1]. The deuteron Zeeman relaxation time describes the relaxation of the magnetization of a quadrupolar nucleus due to fluctuations of the electric field gradient of the chemical bond bearing the resonant nucleus. This is a single-spin description, and is adequate when the dipolar interaction with neighboring spins can be neglected.[2] $T_{1 Q}$ and $T_{1 D}$ refer to the relaxation of quadrupolar or dipolar order created by a pulse sequence (Jeener-Broekaert experiment [27]) that transfers the magnetic Zeeman order to the quadrupolar or dipolar reservoirs. The three first lines of Table 1 represent situations which can be adequately described by a picture of isolated spins, acted on by a random perturbation coming from the neighboring spins. On the contrary, the case of dipolar order relaxation is basically different because in this case the observable $\left\langle\mathcal{H}_{D}^{0}\right\rangle$ involves the interactions among many spins. Here we included the two-spin formula for $T_{1 D}$ reported in the literature. Nevertheless, a recent field cycling study showed that this formula is incomplete for describing the experimental data in nematics [28, 29, 30] (see Section IV).

\section{Applications}

\section{A. The Field-Cycling Technique}

Measurements of the relaxation times $\left(T_{1 Z}, T_{1 D}\right.$ or $T_{1 Q}$ ) provide, in principle, a means of determining the spectral densities of the molecular motions. According to Table I, by combining data of different relax- 
ation experiments, it is possible to determine $J^{1}(\nu)$ and $J^{2}(\nu)$ separately.[28] Then, the comparison of their dependence with the magnetic field and temperature with the theoretically predicted dependence for the involved molecular fluctuations, allows to obtain information about the different motional processes involved. For example, conventional NMR temperature dependent experiments of Zeeman and quadrupolar alignment relaxation in selectively deuterated liquid crystals, allow to characterize intramolecular motions and also give some consistent information about the order fluctuations. [31, 32] The dynamics of the aliphatic chains in nematics was studied in detail by using this method and comparing with theoretical model for molecular potentials.[2, 33]

However, since the conventional NMR studies provide the $T_{1 Z}$ profile only in a rather narrow spectroscopic window, the fitting of data with formula from Table I and Eq.(11) becomes ambiguous and it is difficult to separate the contribution from the different relaxation mechanisms. Due to this weakness, some early high frequency measurements $[34,35,36]$ believed to have found the theoretically predicted square root frequency dependence characteristic of the OFD in nematics [see Eq. (12].

This apparent result is incorrect because the reported fits in the $\mathrm{MHz}$ range do not coincide with subsequent low frequency mesurements when extrapolated. The mistake may be originated in the fact that the other relaxation mechanisms possess a narrow range where the corresponding spectral densities can be approximated by $J(\nu)=a \nu^{-1 / 2}+b$.

A more comprehensive relaxometric study can be performed by extending the measurements to the $\mathrm{kHz}$ domain. However, standard NMR spectrometers cannot perform low frequency measurements, because the signal amplitude strongly decreases at lower external magnetic fields. The adequate experimental method to solve this difficulty is the field-cycling technique, since it allows to enlarge the range of the motional spectrum scanned by longitudinal spin-lattice relaxation experiments, by many orders of magnitude. Details of the basic principles of the technique can be found in Reference [3] and [37] In few words, the method is based on the concept of adiabatic demagnetization. The spin system is polarized at high fields ( frequency in the $\mathrm{MHz}$ re- gion). Then, the external field is adiabatically switched to a selectable lower level. The spin system evolves (relaxes) under these conditions, and after a given interval the field is again adiabatically switched to its original value, where the magnetization is detected by applying a radiofrequency pulse (see Fig. 1a). So doing allows to deal with a $\mathrm{MHz}$ quality signal but now keeping the information of the spin-lattice relaxation at low field.

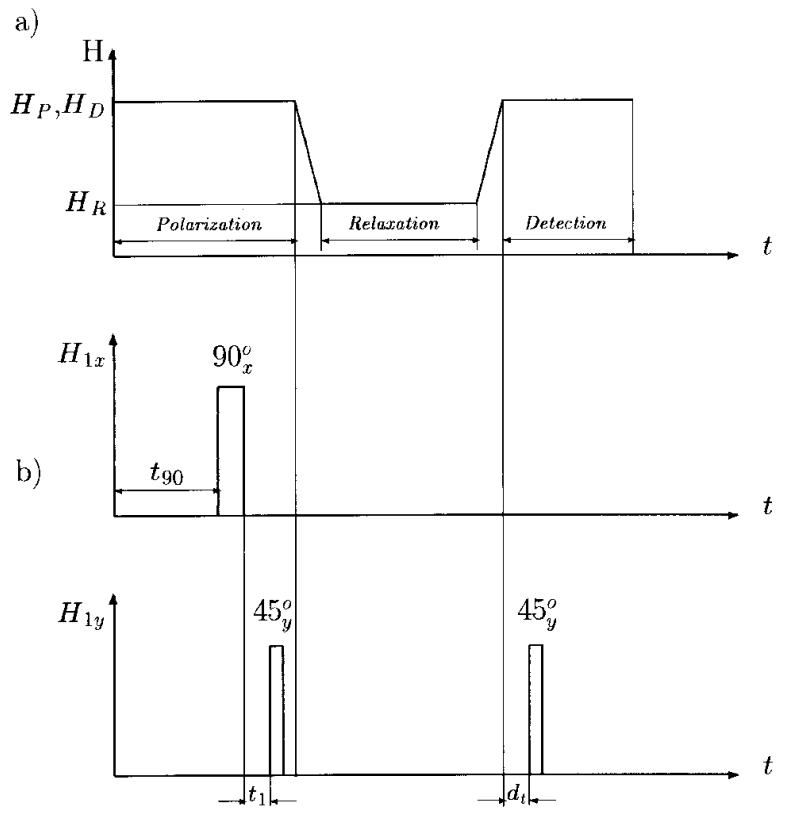

Figure 1. a) Typical modulation of the Zeeman field in a field cycling NMR experiment. b) Scheme of the JeenerBroekaert pulse sequence combined with a field cycling. During the polarization period two phase shifted pulses are applied to create dipolar order. Subsequently the spin system evolves in a different magnetic field during the relaxation period. Finally, after switching back the Zeeman field to the detection value $H_{D}$, a read pulse is applied and the dipolar signal is acquired.

\section{B. Selected Experiments}

A systematic study of the Larmor frequency dependence of $T_{1 Z}$ in thermotropic liquid crystals was carried out mainly by the group of F. Noack (Universitat Stuttgart). Particularly, the nematic phase has been extensively studied. [3, 38, 39, 40]

An illustrative example is the textbook compound PAA (4,4' - dimethyloxyazoxybenzene). Fig. 2 shows the proton relaxation dispersion $T_{1 Z}(\nu)$ for nematic and isotropic phases. The isotropic phase shows the characteristic behavior of common liquids. On the contrary, relaxation in the nematic phase exhibits a well developed $\nu^{1 / 2}$ dependence in the frequency range 
$10^{3}-10^{6} \mathrm{~Hz}$, revealing the occurrence of order fluctuations of the director.

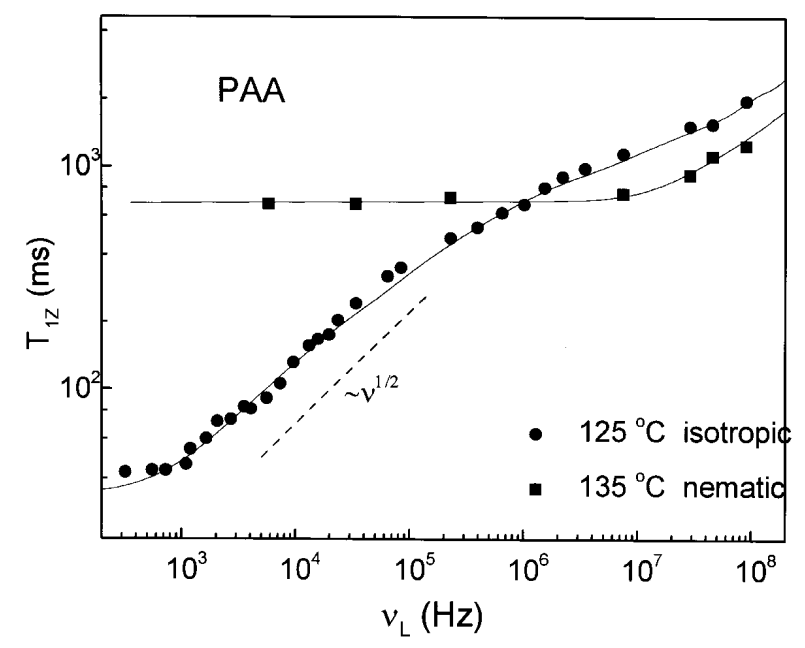

Figure 2. Proton relaxation dispersion $T_{1 Z}(\nu)$ for nematic and isotropic PAA. The nematic phase shows a square root frequency dependence, that is absent in the isotropic phase. The solid lines are model fits including OFD, self-diffusion, molecular rotation and a low frequency cut-off. Data from Ref.39.

Typically, in thermotropic liquid crystals the several mechanisms contributing to spin relaxation can be considered as statistically independent processes. Under this condition, the spectral densities can be calculated as a sum of separate contributions from the molecular rotational tumbling (ROT), traslational selfdiffusion (SD) (local motions) and the OFD:

$$
J^{q}(\nu)=J_{O F D}^{q}(\nu)+J_{S D}^{q}(\nu)+J_{R O T}^{q}(\nu) .
$$

The three spectral densities are:

1. Order Fluctuations of the Director

1a. Nematics $[12,13]$

$$
J_{O F D}^{1}(\nu)=A \nu^{-1 / 2},
$$

with

$$
A=\frac{k_{B} T S^{2}}{2 \pi K r^{6}}\left(\frac{K}{\eta}+D\right)^{-1 / 2} \frac{\left(3 \cos ^{2} \alpha-1\right)^{2}}{4},
$$

where $k_{B}$ is the Boltzmann constant, $K$ is the average Frank constant, $\eta$ is the viscosity, $r$ the interproton distance and $\alpha$ stands for the angle between the internuclear vector and the molecular axis.

1b. Smectics [14]

$$
J_{O F D}^{1}(\nu)=B \nu^{-1},
$$

with

$$
B=\frac{k_{B} T S^{2}}{\pi K_{11} \xi r^{6}} \frac{\left(3 \cos ^{2} \alpha-1\right)^{2}}{4},
$$

where $K_{11}$ is the splay elastic constant (assumed to be equal to the twist constant $K_{22}$ ) and $\xi$ is the coherence length in the direction perpendicular to the smectic layers. The spectral density $J_{O F D}^{2}$ is assumed to be negligibly in the small amplitude approximation. [10]

\section{Rotation[2]}

$$
J_{R O T}^{q}(\nu)=\frac{3}{2} B_{R} \tau_{R} \frac{q^{2}}{1+\left(q \pi \nu \tau_{\perp}\right)^{2}}
$$

where $B_{R}$ is a relaxation amplitude factor depending on both bulk and molecular properties and $\tau_{R}$ is the correlation time of the reorientation about the short molecular axes.

3. Self-Diffusion [6]

$$
J_{S D}^{q}(\nu)=\frac{C \tau_{D} q^{2}}{x^{4}}\left[u+(u \sin x+v \cos x) e^{-x}\right]
$$

where $u=\left(\frac{x}{2}-\frac{1}{x}\right), v=\left(\frac{x}{2}+\frac{1}{x}+2\right)$, and $x \equiv$ $\sqrt{q \pi \nu \tau_{D}}, C$ is the diffusion amplitude factor, and $\tau_{D}$ is the correlation time of the translational molecular jump.

The models described above allow a quantitative description of relaxation dispersion. As an example, Fig. 3 shows the $T_{1 Z}(\nu)$ profile for the nematic $\mathrm{HpAB}$ (4,4' -bis-heptyloxyazoxy-benzene) with the contributions from the different relaxation mechanisms.[28] This is a characteristic profile of thermotropic nematics. It is clear again that the OFD dominate the relaxation in the $K H z$ regime. For the fittings it was also considered a low frequency cut-off $\nu_{c}$ as predicted by R. Blinc.[41] 


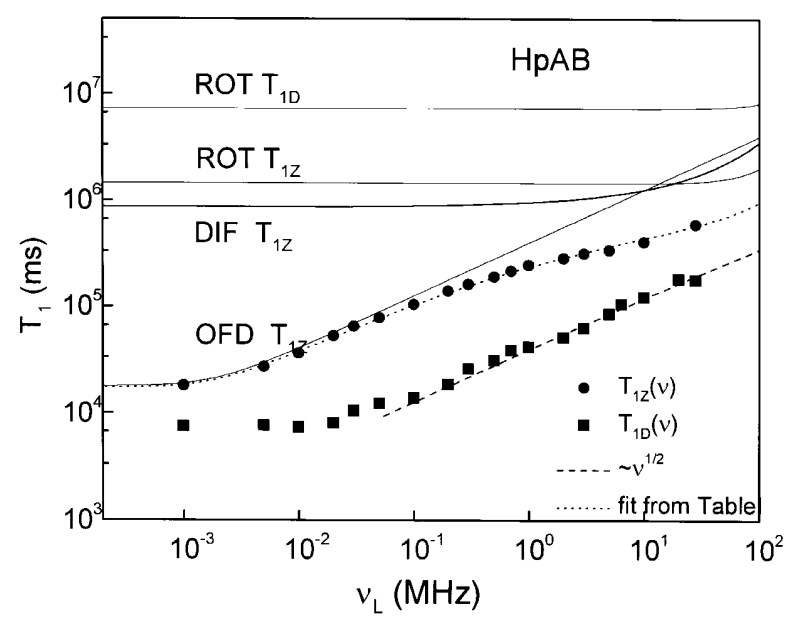

Figure 3. Proton relaxation of the Zeeman and the dipolar order in nematic $\mathrm{HpAB}$ showing the three individual contributions for $T_{1 Z}(\nu)$ together with the rotation term for $T_{1 D}(\nu)$ (self-diffusion does not contribute to dipolar relaxation -see text). Rotations contributes appreciably to Zeeman order relaxation in the $M H z$ regime, but are negligible for dipolar order relaxation in all measured range. Data from Ref.28.

The FC measurements allowed to overcome some ambiguities in the evaluation of the parameters involved in the fittings, namely $A, B, B_{R}, C, \nu_{e}, \tau_{R}, \tau_{D}$. Particularly, the experimental amplitude factor $A$ can be obtained with good precision. The consistency of the fittings is supported by reasonable magnitudes of the parameters and systematic parallels for all the measured compounds. For instance, the fitted amplitude factors $A$ have a good agreement with the theoretical prediction from Eq.(13), for many studied compounds.[3, 42]

Moreover, the obtained values for the self-diffusion correlation time $\tau_{D}$ can be compared with diffusion constant measurements by means of a special combination of NMR Field-Cycling with pulsed field gradients techniques.[43] Assuming the relation [6] $\tau_{D}=\frac{b^{2}}{6 D}$ (with the distance of closest proton approach for diffusion and $D=\frac{1}{3}\left(D_{\|}+2 D_{\perp}\right)$ an effective diffusion constant), the calculated correlation times coincide in order of magnitudes with the fitting parameters. [43]

Another interesting feature arising from FC measurements is the occurrence of the low frequency cutoff typically about few $\mathrm{KHz}$ ( Fig. 3). At the present, two possible explanations for this behavior have been suggested: the first one states that, since the minimum field which could give rise to Zeeman order cannot be less than the local fields produced by the neighboring spins, it would be expectable that for frequencies of the order of few $\mathrm{KHz}, T_{1 Z}$ became frequency independent; the second one refers to the finiteness of the nematic order correlation length, [41] $\xi$, due to the presence of disclinations and other defects. In such a case only order fluctuations with a wavelength smaller than $\xi$ can take place, giving rise to a minimum cut-off frequency, through the 'dispersion relation'

$$
\nu_{c}=\frac{2 \pi K}{\eta \xi^{2}}
$$

where $K$ is the effective elastic constant and $\eta$ is the effective viscosity. Although most workers favor the second hypothesis, a final experimental confirmation is still lacking and the subject is nowadays being investigated theoretically and experimentally.[44]

The thermotropic smectic mesophase has not been studied so extensively as the nematic one. Nevertheless, the available experimental results show clearly the presence of collective motions. $[19,45]$ Fig. 4 shows the $T_{1 Z}$ profile for TBBA in the smectic A phase.[19] It can be appreciated the predicted $\nu^{1}$-law for order fluctuations within a smectic layer [Eq.(14)]. This linear dependence is evident in the frequency range $10^{3}-10^{5} \mathrm{~Hz}$, showing again that the OFD are the dominant relaxation mechanism for the Zeeman order only at low Larmor frequencies. Two additional relaxation mechanisms (selfdiffusion and anisotropic rotations) are necessary to describe the experimental results.

Recently, other workers [46] have measured a transition from $\nu^{1}$ to $\nu^{1 / 2}$ in the $T_{1 Z}$ frequency dependence of smectic $8 \mathrm{CB}$ (4- cyano-4' -8-alkylbiphenyl) at $23^{\circ} \mathrm{K}$, i.e. close to the smectic A-nematic transition. A similar result was observed in the smectic $\mathrm{HpAB}$ at $82^{\circ} \mathrm{K}$, but now far from the transition. [47] Conceivably, this feature was not detected in earlier measurements due to the low density of experimental points. At the present, it is not clear if this is a pretransitional effect or if it reflects an intrinsic characteristic of the collective fluctuations in the smectic phase. Evidently, more systematic measurements are needed. 


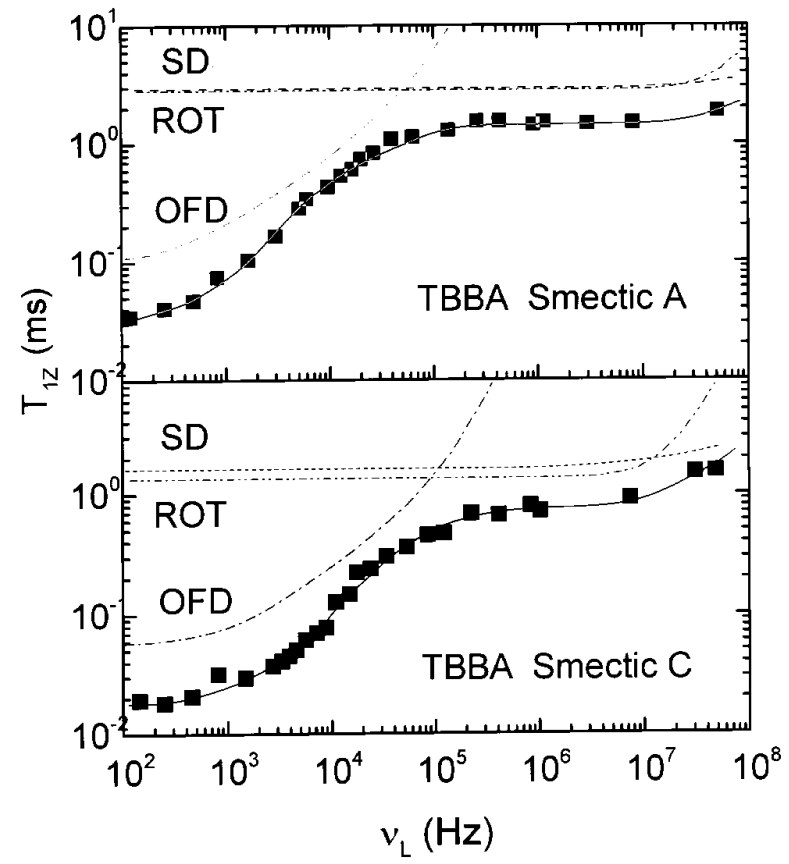

Figure 4. Proton spin relaxation dispersion, $T_{1 Z}(\nu)$, for TBBA in smectic $\mathrm{A}$ and smectic $\mathrm{C}$ phases. The solid line is the fit according to Table I and Eq. (11) including again OFD, molecular rotations and self-diffusion. Data from Ref. 19 .

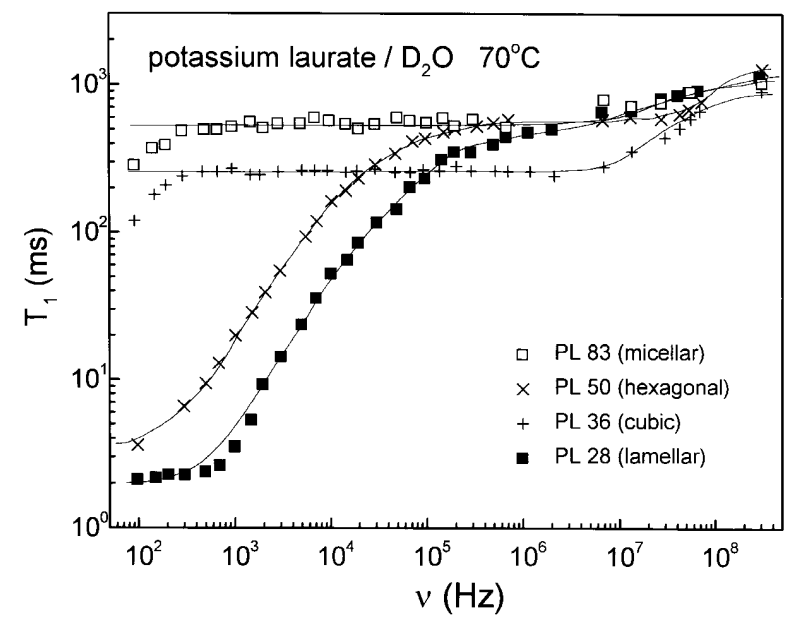

Figure 5. Proton relaxation dispersion $T_{1 Z}(\nu)$ for the micellar, hexagonal, cubic and lamellar phase of a lyotropic potassium laurate- $\mathrm{D}_{2} \mathrm{O}$ mixtures. The micellar and the hexagonal system show the $\nu^{1}$ dependence typical of smectics. The linear profile is absent in the isotropic phases. Data from Refs. 3,18 .

The linear frequency dependence is better developed in lyotropic liquid crystals. As pointed by Kuhner et $a l$, [48] this feature is expectable because the smectic type layers are almost decoupled by the water interface. In this way, each one constitutes basically a two-dimensional system. An example are lamellar or hexagonal potassium laurate-water mixtures (see Fig.
$5)$, where the $\nu^{1}$-law is observed approximately in the range $10^{3}-10^{5} \mathrm{~Hz}$.

An independent experiment providing additional experimental evidence of the presence of collective fluctuations in nematics are NMR field-cycling studies of deuteron spin-lattice relaxation. Contrarily to proton NMR spectra, the deuteron spectra show without any spin decoupling technique many well-resolved line doublets, which can be assigned to non-equivalent sites on the molecule. [17] At present it was not yet attained enough signal quality and resolution to separate the corresponding relaxation times for all the observed doublets, due to the unfavorably smaller gyromagnetic ratio (a higher detection field is needed). Nevertheless, it is possible to measure the spin lattice relaxation rates for some average spin positions, namely for the ring window and the chain window as shown in Fig. 6. It is interesting to note that, while the dispersion profile of the chain deuterons reveal an approximate square-root law in the same frequency range than the proton data, the rings deuterons exhibit a weak frequency dependence. This fact can be qualitatively understood by realizing that, since the relevant orientation for quadrupolar coupling is the angle between the carbon-hydrogen bond and the static magnetic field, the contribution from the molecular rotations along the long axis of the molecule is significantly greater for deuteron than for proton spin relaxation.[17]

Finally, in Fig. 6 we also show a comparison between proton $T_{1 Z}$ of the nematic 5CB (4- cyano-4' -5 alkylbiphenyl) and the alkyl chain deuteron $T_{1 Z}$ for the per-deuterated nematic $5 C B-d_{19}$. As can been clearly seen the collective motions dominate in coincident frequency ranges.

\section{Dipolar order relaxation}

Field Cycling experiments are able to reveal the frequency range where cooperative motions dominate relaxation as well as giving an approximate value for the relative weight of the different relaxation mechanisms in a number of nematics and smectics.

The NMR proton spectra of liquid crystals generally present unresolved broad lines, due to the strong dipolar interactions. The measured relaxation times represent all spins in the molecule, and the contributions to the spectral densities from different kinds of sites are superimposed. When the molecular motions are complex, $T_{1 Z}(\nu)$ alone is insufficient for disentangling the 
different contributions to the spectral densities. Then it would be adequate to complement the data of Zeeman relaxation with another experiment. Under the assumption that the (weak order) formulae from Table I hold in the whole frequency range, measuring the frequency dependence of different relaxation times, like $T_{1 D}$ (or $T_{1 Q}$ ) together with $T_{1 Z}$ comes out to be a more descriptive experiment. The spectral density $J^{1}(\nu)$ can be directly determined through the first one and then, by using it in the formula for the latter, $J^{2}(2 \nu)$ can be calculated.

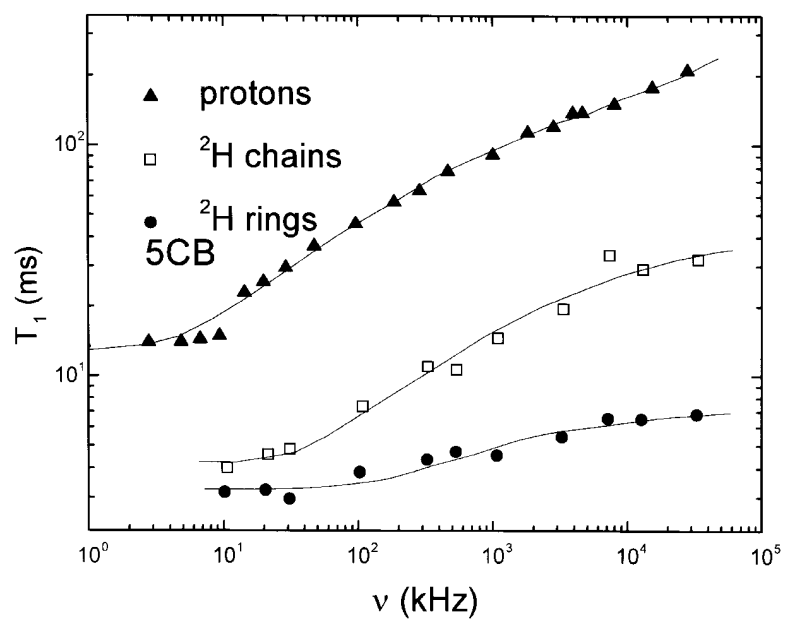

Figure 6. Comparison of the frequency dependence of $T_{1 Z}$ in protons of $5 \mathrm{CB}$ (triangles), chain deuterons of $5 \mathrm{CB}-d_{19}$ (open squares) and ring deuterons only (circles). Deuteron measurements allow to select a separate frequency window for different sites in the molecule. Data from Ref. 17.

The first step in this direction was the field cycling measurement of both proton $T_{1 Z}(\nu)$ and $T_{1 D}(\nu)$ in a family of thermotropic nematic liquid crystals.[28, 40] This method for measuring $T_{1 D}$ combines the field cycling technique with the Jeener-Broekaert pulse sequence for creating dipolar order. Figs. (1b) and (1c) show a diagram of the technique. Fig. (3) shows the dispersion of $T_{1 D}$ and $T_{1 Z}$ in the nematic phase of HpAB. The first outstanding feature is that $T_{1 D}(\nu)$ follows the typical trend of the OFD in the whole frequency range (even for $\nu \gg 10 \mathrm{MHz}$ !). This behavior is very different from that of $T_{1 Z}$ where, as a rule, the OFD dominate, at most up to hundreds of kilohertz. Another interesting feature is the occurrence of a cutoff frequency about $5 \times 10^{4} \mathrm{~Hz}$, which is an order of magnitude higher than the one of Zeeman relaxation time.

The two-spin model for dipolar relaxation (see Table 1) predicts that $T_{1 Z} / T_{1 D} \leq 3$ over all the frequency range, since the spectral densities are positive quantities. Conversely, the experiments show that this ratio is greater than three in both $\mathrm{HpAB}$ and $8 \mathrm{CB}$, as displayed in Fig. 7. Also, as can be seen in Fig. 8, there is a noticeable gap between the $T_{1 D}$ calculated with the isolated phenyl spin pairs model and the experimental data; the difference is frequency dependent and proportional to $\nu^{-1 / 2}$, even at high frequencies. Similar characteristics were found in other several compounds in the nematic phase, $[29,40]$ where it was realized that the gap increases with the size of the molecule.
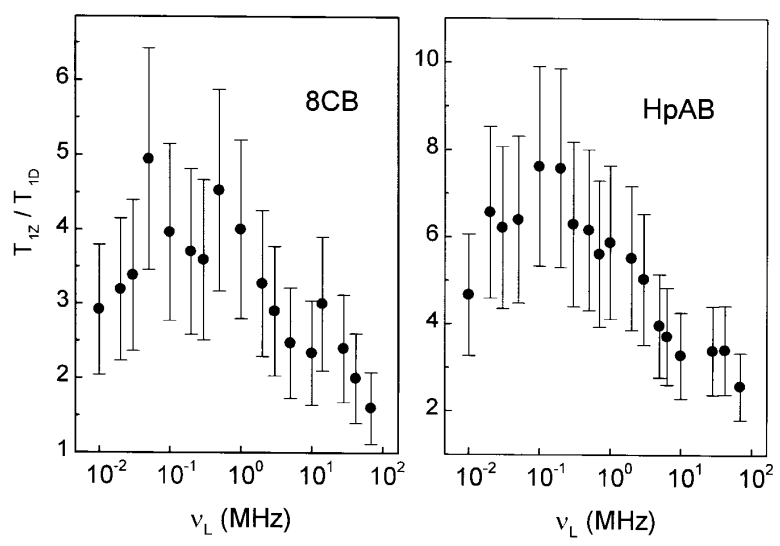

Figure 7 . The quotient $T_{1 Z} / T_{1 D}$ as a function of the Larmor frequency in the nematic phase of $\mathrm{HpAB}$ and $8 \mathrm{CB}$. This ratio exceeds the value of three in discrepancy with the semiclassical two-spins model. Data from Ref.28.

As can be seen in Fig.(3), the contribution from the OFD to $T_{1 Z}$ decays rapidly for increasing external field, and it generally becomes comparable to the spectral densities of rotational and translational diffusion for frequencies higher than $5 \times 10^{5} \mathrm{~Hz}$. The situation is rather different for dipolar relaxation, since it is mainly driven by the OFD mechanism even at high fields. It can be clearly appreciated in Fig.(3) the larger influence of the OFD as compared with the molecular rotational diffusion. It can be affirmed that the rotations are practically negligible in $T_{1 D}$.

These experimental findings are challenging since they clearly point out some deficiencies of the existent theoretical approach, but, also reveal $T_{1 D}(\nu)$ as a magnitude that reflects the OFD almost exclusively in the available frequency range.

The fact that $T_{1 Z} / T_{1 D} \geq 3$ indicates that the observed dipolar order relaxation is faster than the one predicted by the traditional model. On the contrary 
$T_{1 Z}(\nu)$ is well described by the formula from Table I in numerous liquid crystals in the high field limit $\left(H_{0}>H_{\text {local }}\right) .[2,3,31,32,49]$ Therefore, the failure was assigned [28] to the standard two-spin (phenyl) approach for dipolar order spin-lattice relaxation rate.

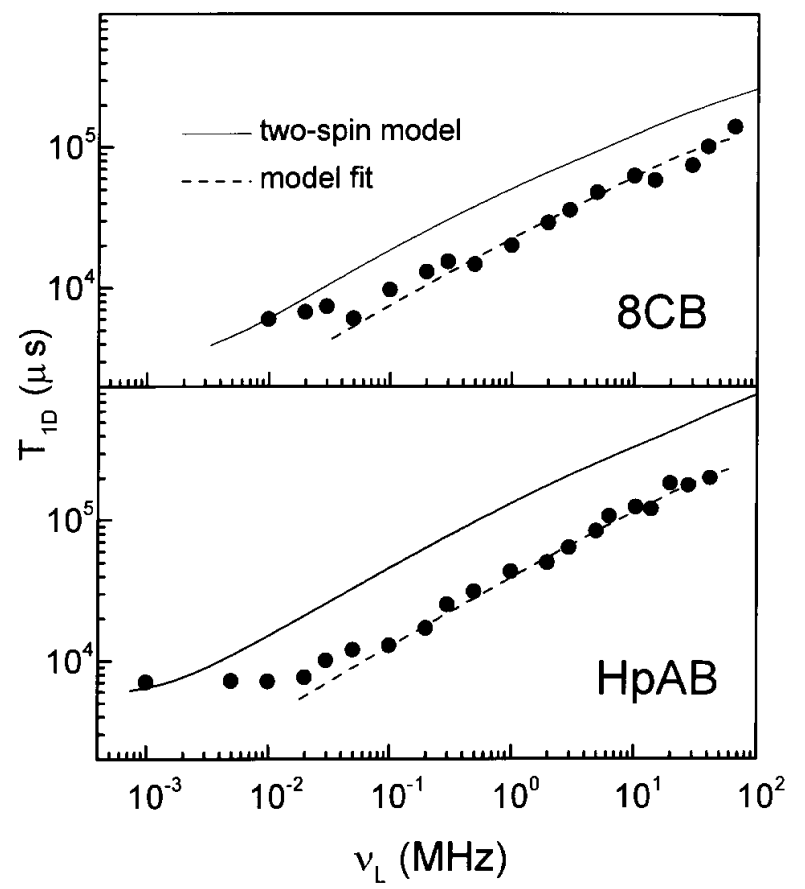

Figure 8. Frequency dependence of the dipolar order relaxation in the nematic phase of $\mathrm{HpAB}$ and $8 \mathrm{CB}$. The full line corresponds to the prediction from the two-spin model and the dashed line is the best fit with the function $T_{1 D}^{-1}=3 C_{D} J^{1}(\nu)_{\text {intra }}+a \nu^{1 / 2}+b$. Data from Ref. 28 .

It should be kept in mind that the usual model for dipolar relaxation in thermotropic liquid crystals[14, 50] starts from the weak order master equation [Eq.(9)] and considers the spin system as an ensemble of isolated spin pairs. Only intramolecular contributions are kept, arguing that the rapid diffusive molecular motion averages out the intermolecular contribution. The chain protons are not considered either, due to their high mobility. Another important hypothesis is the one known as 'fast motion', that is $\nu_{D} \tau_{c} \ll 1\left(\nu_{D}\right.$ a typical dipolar frequency and $\tau_{c}$ a lattice correlation time). This condition allows to neglect the spin-spin interactions in calculating the time evolution of the spin operators in the interaction picture. [5]

The observed frequency dependent gap between theory and experimental data indicates that the model for $T_{1 D}$ in liquid crystals is oversimplified and has neglected important mechanisms of relaxation, driven by the OFD. For instance, keeping only two spins avoids the presence of spectral densities evaluated at zero frequency or frequencies of the order of magnitude of the dipolar coupling, $J^{0}\left(\nu_{D}\right)$, because the coefficients associated to these terms involve spin traces which are identically zero for two spins. The physical reason of this is that these coefficients represent 'flip-flop' transitions, that do not involve changes in the dipolar energy of only two spins. Also spectral densities with $q=2, J^{2}(2 \nu)$, do not appear due to similar reasons. According to the proton spectra in nematics, there is an appreciable dipolar contact among the core and chain protons, giving the possibility of some effects associated to interpair interactions. This imposes a revision of the validity of the hypothesis involved in the model for dipolar relaxation.

A partial answer came after a recent experimental and theoretical investigation of $T_{1 Z}(\nu)$ and $T_{1 D}(\nu)$ in PAA and in the methyl-deuterated PAA $d 6 .[29,51]$ Deuterating the methyl chain yields a proton system reflecting only the dynamics of the core. The frequency dependence of the relaxation times in both compounds showed discrepancies with the two-spin theory, similar to those previously observed in $\mathrm{HpAB}$ and $8 \mathrm{CB}$. Fig. (9) shows the obtained results for the frequency dependence of $T_{1 Z}$ and $T_{1 D}$ at $130^{\circ} \mathrm{C}$. The figures also include a plot of the underestimated dipolar relaxation rate as given by Table 1 . The experimental data of the dipolar relaxation time can be well represented by the function $T_{1 D}^{-1}=3 C_{D} J^{1}(\nu)_{\text {intra }}+a \nu^{-1 / 2}+b$, down to $10^{5} \mathrm{~Hz}$. The first correction term has the well known frequency dependence of the OFD, [12] and the parameter $a$ is $5.3 \times 10^{3} s^{-3 / 2}$ and $4 \times 10^{3} s^{-3 / 2}$ for $P A A$ and $P A A_{d 6}$, respectively. The frequency independent term is related to the spectral densities with $q=0$ which appear if the model of isolated spin pairs is abandoned. In this case, the constant are mainly associated to the dipolar interaction between the chain and the core. This term is not dominant $\left(b=0.7 \times s^{-1}\right.$ for $P A A$ and $b=0.2 s^{-1}$ for $\left.P A A_{d 6}\right)$, and contribute appreciably only in the $M H z$ range. For instance, at $10 \mathrm{MHz}$, the constant term represents the $7 \%$ of the total relaxation rate for $P A A_{d 6}$.

In relation with the theory, following the lines of the semiclassical formalism of spin-lattice relaxation[5] a general high field expression for $T_{1 D}^{-1}$ in nematic liquid crystals valid for an arbitrary number of spins was 
deduced giving the result: [29]

$$
\begin{aligned}
T_{1 D}^{-1}= & T_{1 D c o r r}^{-1}+\frac{27 \gamma^{4} \hbar^{2}}{8 N} \sum_{l k} J_{l k}^{1}(\nu) \frac{{\overline{F_{l k}^{0}}}^{2}}{\sum_{j}{\overline{F_{l j}^{0}}}^{2}}+\frac{4 \gamma^{4} \hbar^{2}}{3 N \sum_{j}{\overline{F_{l j}^{0}}}^{2}} \sum_{l k} \sum_{q} J_{l k}^{q}(\nu q) \times \\
& \left\{a^{q} \sum_{m \neq k}\left({\overline{F_{l m}^{0}}}^{2}+{\overline{F_{k m}^{0}}}^{2}\right)+b^{q} \sum_{m \neq k} \overline{F_{l m}^{0}} \overline{F_{k m}^{0}}\right\}
\end{aligned}
$$

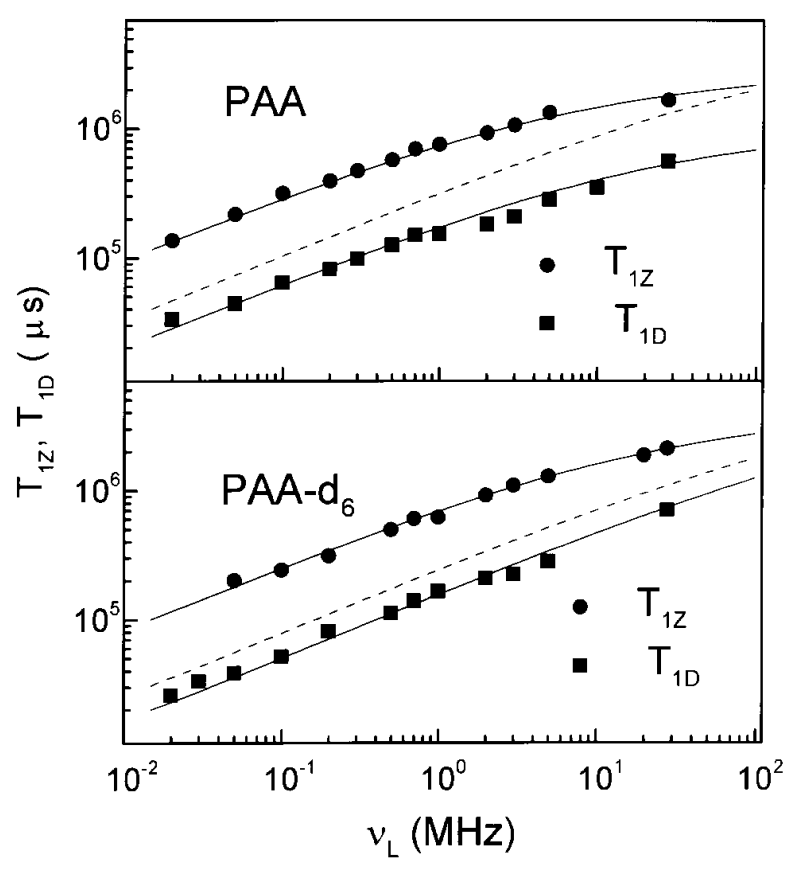

Figure 9: Zeeman and dipolar relaxation rates, measured with the field cycling technique in nematic $P A A$ and nematic $\mathrm{PAA}_{d 6}$ at the same temperature. The circles and squares are experimental $T_{1}$ and $T_{1 D}$ respectively. The upper full lines are the fitting to $T_{1 Z}$ with Table I and Eq.(11). The dashed lines are the semiclassical two-spin model for $T_{1 D}$ from Table I. The lower full lines are the best fits with the function $T_{1 D}^{-1}=3 C_{D} J^{1}(\nu)_{\text {intra }}+a \nu^{1 / 2}+b$ (see the text). Data from Ref.29.

The first term corresponds to interpair cross correlations, while the others involve uncorrelated interactions. All terms have similar structure: they are proportional to angular averages of the type ${\overline{F_{l j}^{0}}}^{2}$. The intermolecular contributions cancel due to the rapid relative motion of the molecule. Accordingly, the translational self-diffusion does not contribute appreciably to dipolar order relaxation in nematics.

This general result allows to investigate the role of interpair correlation of protons of the core and the chains. When particularized to $\mathrm{PAA}_{d 6}$ for calculating the contribution to $T_{1 D}$ by including second neighbor protons, the calculation yielded a negligible correction to the usual two-spin prediction. This fact, which is consequence of the $r^{-3}$ dependence of the dipolar energy interaction, is consistent with NMR line shape second-moment studies[52] that characterize $\mathrm{PAA}_{d 6}$ as a two-spin system.

According to the former results, the dipolar order relaxation of $\mathrm{PAA}_{d 6}$, although seeming to be a two-spin problem, cannot be described by the usual formula for isolated spin pairs!. This means that the observed gap has to be associated to some other type of processes which are not considered by this approach.

A point still requiring discussion is the fast motion hypothesis, contained in the basic weak-order theory. Technically, this amounts to calculating the evolution of the spin operators in the interaction picture with the operator $\exp ^{i t / \hbar \mathcal{H}_{z}}$ (a simple rotation around the direction of the magnetic field). For the slowest components of the OFD the condition $\nu_{D} \tau_{c} \ll 1$ is not fulfilled; then neglecting the spin interactions during the lifetime of the fluctuations may be inadequate. In such cases, the time evolution has to be calculated using the complete operator $e^{i t / \hbar\left(\mathcal{H}_{Z}+\mathcal{H}_{D}\right)}$ in order to the introduce the interactions. However, this correction to the standard approach did not introduce significant changes either.[30] That is, this procedure does not lead to a frequency dependent correction as needed for explaining the $T_{1 D}(\nu)$ dispersion.

The insensibility shown by the weak-order result to the inclusion of the spin interactions in the microscopic time scale, can be interpreted as follows: As shown in Section II, the weak order master equation is obtained by neglecting terms of quantum mechanical character in Eq.(8). The relaxation rates calculated in this limit are formally equivalent to those calculated directly from 
a stochastic Liouville equation, where the lattice variables of the spin-lattice Hamiltonian are given random time functions. By representing the lattice variables with arbitrary random functions of time, the backreaction of the lattice on the spin system during the lifetime of the fluctuations cannot be retained.

In summary, the former example allows to safely conclude that the weak-order theory does not contain all the ingredients necessary to explain the dipolar order relaxation in systems having slow fluctuations, like nematic thermotropics. According to this outstanding conclusion, the failure must be sought in the very basic assumptions of the weak-order relaxation theory.

In order to include the correlation between a many body system and the lattice in the microscopic time scale, it is necessary to keep the quantum terms of Eq.(8). Namely, when dealing with the relaxation of the dipolar order, the spin system should be treated as an open quantum system in thermal contact with a quantum mechanical lattice. This can be done by eliminating the weak-order assumption.[30, 53] Under this general condition, the master equation in the form of Eq.(8) is not easily applicable to the calculation of the time dependence of spin observables. A more tractable equation follows by performing a series expansion of the master equation in operator form. [54] The lowest order term of the expansion coincides with the first term of Eq.(8), while the higher order ones represent the quantum mechanical part. In fact, the new contributions cancel if the lattice is represented by a stochastic process. The formalism is, at this point, adequate for introducing a 'trial' solution. The trial density operator commonly used in NMR is the one corresponding to the spin temperature assumption. Physically, it consists in assuming that the relaxation can be described as a succession of semi-equilibrium states.[55, 56] Doing this, it is straightforward to calculate a relaxation time in terms of the spectral densities.[30]

Using the Pincus-Blinc model for the nematic hydrodynamic motions, leads to a correction term to the usual expression of the dipolar order relaxation rate, having the form $a \nu^{-1 / 2}$. This result agrees with the phenomenological expression used in fitting $\mathrm{PAA}_{d 6}$ and PAA data, and other thermotropic nematic liquid crystals. Accordingly, the discrepancy between experiments and the weak order theory can be explained in terms of multispin processes having a quantum mechanical character.

The existence of the anomalous low-frequency cutoff can also be discussed in terms of the quantum mechanical approach. When the external magnetic field is lowered so that the Larmor frequency is comparable with the linewidth $\left(10^{5} \mathrm{~Hz}\right)$, the description is made in terms of the dipolar and not the Zeeman energy levels. Under this condition the Larmor frequency ceases to be a collective parameter of the spin system.

\section{Final comments}

Frequency dependent $T_{1 Z}$ experiments using the field cycling technique have shown useful in characterizing the OFD. This is specially clear in nematics, where the typical frequency behavior dominates in a broad frequency range and less evident in smectics. The fact that smectics have slower local molecular motions than nematics has a double effect in reducing the effectiveness of $T_{1 Z}(\nu)$ for displaying unambiguously the contribution of the collective motions. On the one hand individual molecular motions (rotation and diffusion) have a greater relative weight in smectics. On the other, the frequency dependence of the OFD is steeper. This amounts in a narrowing of the frequency range where the OFD dominate relaxation [see Fig.(4)].

Due to the strong spin-spin interaction, the proton Zeeman relaxation time is an average parameter representing all the protons placed in different kind of sites (for example in the chain or the core). In molecules having many protons this produces a certain degree of ambiguity in the physical parameters obtained from the fittings, due to the large number of parameters that have to be determined. More informative nuclei would be deuterons (D) or carbons $\left({ }^{13} \mathrm{C}\right)$ because they separate individual atomic sites on the molecules by a generally well-resolved spectrum. However, such experiments imply important technological difficulties. This limitation can be partially eliminated, in a more accessible way by combining experiments of relaxation in protons of partially deuterated molecules; so doing the different relaxation mechanisms can be separated by alternatively deuterating the chains or the cores. Also, these experiments can be complemented with angular 
dependent measurements, which provide additional experimental information $[14,22,57]$

In Section (IV) we showed some examples where dipolar order relaxation reflects the OFD almost exclusively. This outstanding feature makes $T_{1 D}(\nu)$ an optimum parameter for studying slow collective molecular motion in mesophases. Accordingly, it could be helpful for testing theoretical models for the hydrodynamic fluctuations in mesophases having different dimensionality.

Due to the different frequency response of both relaxation times, experiments of $T_{1 D}(\nu)$ in conjunction with $T_{1 Z}(\nu)$ may provide valuable experimental tools for the study of molecular motions in mesophases. Particularly, it would allow to clearly discern the frequency dependence of the collective motions in smectics. However, for these studies to be fruitful, a thorough theoretical revision of the effect of the slow fluctuations of the director on the dipolar relaxation rate becomes necessary, since a comprehensive expression of $T_{1 D}^{-1}$ in terms of spectral densities is still lacking.

Parallel to the field of the applications, from a basic perspective, dipolar order relaxation also provides an interesting example of irreversible processes where the spin-spin interactions occurring during the microscopic time intervals seem to produce observable effects in the transport parameters.

\section{Acknowledgment}

We are grateful to Dr. D. J. Pusiol for helpful discussions. This work was partially supported by CONICOR and SECYT from Córdoba, CONICET and Fundación Antorchas from Argentina. The authors dedicate the present work to the memory of Prof. F. Noack who made important contributions to the field of NMR in mesophases.

\section{References}

[1] A.Abragam, The Principles of Nuclear Magnetic Resonance(Oxford U.P. London 1961), Chap. VIII.

[2] R.Y. Dong, Nuclear Magnetic Resonance of Liquid Crystals (Heidelberg, Springer, 1994).

[3] F. Noack, M. Notter, and W. Wiess, Liq. Cryst. 3, 907 (1988).

[4] J.M.Deutch and I. Oppenheim, Adv. in Magnetic Resonance, 3, 43 (1968).
[5] J. Jeener, Advances in Magnetic Resonance 3, 205 (1968).

[6] M. Vilfan and S. Zumer, Phys. Rev. A 17, 424, (1978).

[7] M. Vilfan and S. Zumer, Phys. Rev. A 21, 672 (1980).

[8] D. Woessner, J. Chem. Phys. 37, 647 (1962).

[9] P. DeGennes and J. Prost, The Physics of Liquid Crystals 2 nd Edition (Oxford, Clarendon Press, 1993).

[10] J.H. Freed, J.Chem. Phys. 66, 4183 (1977).

[11] G.P. Zientara and J.H. Freed, J. Chem. Phys. 79, 3077 (1983)

[12] P. Pincus, Solid State Commun. 7, 415 (1969).

[13] R. Blinc, D. L. Hogenboom, D. E. O’Reilly, and E. M. Peterson, Phys. Rev. Lett. 23, 17, 969 (1969).

[14] R. Blinc, M. Luzar, M. Vilfan and M. Burgar, J. Chem. Phys. 63 (8), 3445 (1975).

[15] M. Vilfan, M. Kogoj and R. Blinc, J. Chem. Phys. 86, 1055 (1987).

[16] K.H. Schweikert and F. Noack, Mol. Crys. Liq. Crys. 212, 33 (1992).

[17] R. Köllner, K.H. Schweikert, and F. Noack, Liq. Cryst. 13, (4) 483 (1993).

[18] W. Kuhner, E. Rommel, F. Noack and P. Meier, Z. Naturforsch., 42a, 127 (1987).

[19] T. Mugele, V. Graf, W. Wölfel, F. Noack, Z. Naturforsch, 35a, 924 (1977).

[20] E. Rommel, F. Noack, P. Meier and G. Kothe, J. Phys. Chem. 92, 2981 (1988).

[21] J. Struppe, F. Noack and G. Klose, Z. Naturforsch. 52 a, 681 (1997).

[22] J. Struppe and F. Noack, Liq. Cryst. 20, 595-606 (1996).

[23] K. Blum, Density Matrix Theory and Applications, (Plenum Press, New York, 1981).

[24] A. G. Redfield, Adv. Mag. Res. I, p.1 (1965).

[25] J.P. Jacobsen, H.K. Bildsoe and K. Schaumburg, J. Magn. Res. 23, 153 (1976).

[26] H.W. Spiess, J. Chem. Phys. 72, 6755 (1980).

[27] J. Jeneer and P. Broekaert, Phys.Rev 157, 232 (1967)

[28] R. Zamar, E. Anoardo, O. Mensio, D. Pusiol, S. Becker and F. Noack, J. Chem. Phys. 109, 3, 1120 (1998).

[29] O. Mensio, R.C. Zamar, D.J. Pusiol and S. Becker, (submitted)

[30] R.C. Zamar and, O. Mensio, (unpublished).

[31] Nuclear Magnetic Resonance of Liquid Crystals edited by J.W. Emsley (NATO ASI Series, 1985).

[32] R.L. Vold, W.H. Dickerson and R.R. Vold, J. Mag. Reson. 43, 213, (1981). 
[33] R.R. Vold and R. L. Vold, J. Chem Phys. 88, 1443 (1987).

[34] J.W. Doane, J.J. Visintainer, Phys. Rev. Lett. 23, 1421 (1969)

[35] M. Weger and B. Cabane, J. Phys. 30, C4-72 (1969).

[36] M. Vilfan, R. Blinc, and J. W. Doane, Solid St. Commun. 11, 1073 (1972).

[37] F. Noack, in Encyclopaedia of NMR, edited by D.M. Grant and R.K. Harris, Wiley, New York, pp 19801990 (1995).

[38] V. Graf, F. Noack and M. Stohrer, Z. Naturforsch. 32a, 61 (1977).

[39] W, Wölfel, F. Noack, M. Stohrer, Z. Naturforsch 30a, 437 (1975).

[40] S.A. Becker, Thesis, Physikalisches Institut der Universität Stuttgart, (1996).

[41] R. Blinc, NMR Summer School, Waterloo, p.97 (1975).

[42] M. Notter, Diplomarbeit, Physikalisches Institut der Universität Stuttgart, (1986).

[43] J. Mager, Thesis, Physikalisches Institut der Universität Stuttgart, (1993).

[44] F. Vaca Chávez and D. J. Pusiol, "XXI Encontro Nacional de Física da Materia Condensada", Caxambu, Brasil, pag. 179, (1998).

[45] D. Pusiol and F. Noack, Liq. Crys. 5, 377, (1989).
[46] D. J. Pusiol and R. Acosta, private communication.

[47] P. Zollino, Diplomarbeit, Physikalisches Institut der Universität Stuttgart, (1992).

[48] W. Küner, E. Rommel, F. Noack, Z. Naturforsch 42a, 127 (1986).

[49] R. Kimmich, Tomography, Diffusometry, Relaxometry (Berlin, Springer, 1998).

[50] R.G.C. Mc. Elroy, R.T. Thompson and M.M. Pintar, Phys. Rev. A 10, 403 (1974).

[51] O. Mensio, R. C. Zamar, D. J. Pusiol and S. Becker ' $13^{\text {th }}$ ISMAR Satellite Meeting of the Ampere Congress', Berlín, Alemania (1998).

[52] R.Y. Dong, B. Nakka and E. Tomchuk, Mol. Cryst. Liq. Cryst. 38, 53 (1977).

[53] O. Mensio y R.C. Zamar, Anales de la AFA, vol. 8, 138 (1996).

[54] O. Mensio y R. Zamar, "III Workshop Iberoamericano de Fluidos Complejos y sus Aplicaciones", Concepción, Chile (1997).

[55] M. Goldman, Spin Temperature and Nuclear Magnetic Resonance in Solids (Pergamon Press, Oxford 1979).

[56] L.C. Hebel, Solid. St. Phys. 15, 409 (1963).

[57] R.Y. Dong and J. Sandeman, J. Chem. Phys. 78, 4649 (1983). 